IMA Journal of Management Mathematics

Volume 27, Issue 2, 1 April 2016, Pages 235-253

\title{
Corruption, evasion and environmental policy: A game theory approach (Article)
}

Cerqueti, R. ${ }^{3} \otimes$, Coppier, R. $\stackrel{\circ}{\circ}$

aniversity of Macerata, Department of Economics and Law, Via Crescimbeni 20, Macerata, Italy

${ }^{b}$ Department of Economics and Law, University of Macerata, Italy

Abstract

$\checkmark$ View references $(41$

This paper deals with the interaction between polluting firms, tax inspectors and politicians in a corrupted context. We construct a theoretical game model with incomplete information to discuss the effects of such interaction on environmental policy. In this respect, we believe that the State may pursue environmental protection by employing two alternative strategies: on the one hand, the State can, through greater incentive for the tax inspector, increase the monitoring level that reduces the evasion and thus increase tax revenues (incentive channel); on the other hand, the State can, through greater environmental expenses, increase the compliance of the polluting firm which means lower evasion and, thus greater tax revenues (compliance channel). Clearly, more environmental expenses mean, ceteris paribus, less public resources for the tax inspector's incentive, and vice versa. In this context, we demonstrate that, for a country with a high (low) level of incentives, the incentive (compliance) channel is more efficient than the compliance (incentive) channel. (C) 2014 The authors.

\section{Author keywords}

corruption environmental policy Game theory
Indexed keywords
Engineering controlled terms: Crime Game theory Taxation
Compendex keywords $\quad$ Ceteris paribus corruption Environmental expense Environmental policy Game models Incomplete information
Englic resources Tax revenue
Envineering main heading: $\quad$ Environmental protection

ISSN: $1471678 \mathrm{X}$

CODEN: IJMMC

Source Type: Journal

Original language: English
DOI: 10.1093/imaman/dpu019

Document Type: Article

Publisher: Oxford University Press 
IMA Journal of Management Mathematics (2016) 27, 235-253

doi:10.1093/imaman/dpu019

Advance Access publication on 16 October 2014

\title{
Corruption, evasion and environmental policy: a game theory approach
}

\author{
Roy Cerqueti* \\ University of Macerata, Department of Economics and Law, Via Crescimbeni, 20-62100 Macerata, \\ Italy \\ *Corresponding author: roy.cerqueti@unimc.it \\ AND \\ RAFFAELla COPPIER \\ Department of Economics and Law, University of Macerata
}

[Received on 21 September 2013; accepted on 12 September 2014]

\begin{abstract}
This paper deals with the interaction between polluting firms, tax inspectors and politicians in a corrupted context. We construct a theoretical game model with incomplete information to discuss the effects of such interaction on environmental policy. In this respect, we believe that the State may pursue environmental protection by employing two alternative strategies: on the one hand, the State can, through greater incentive for the tax inspector, increase the monitoring level that reduces the evasion and thus increase tax revenues (incentive channel); on the other hand, the State can, through greater environmental expenses, increase the compliance of the polluting firm which means lower evasion and, thus greater tax revenues (compliance channel). Clearly, more environmental expenses mean, ceteris paribus, less public resources for the tax inspector's incentive, and vice versa. In this context, we demonstrate that, for a country with a high (low) level of incentives, the incentive (compliance) channel is more efficient than the compliance (incentive) channel.
\end{abstract}

Keywords: game theory; corruption; environmental policy.

\section{Introduction}

Evidence suggests that the environment is affected by several economic factors. A wide number of authors focus on the impact of energy consumption, technological processes and, in general, human activities on pollution and natural resources (see, e.g., Tapiero, 2009; Bosetti et al., 2002; Cerqueti, 2013 and references therein). In this respect, a relevant role against environmental protection is played by illegal behaviours, with a specific mention to corruption. In general, corruption represents one of the most acknowledged enemies of the development of a country. Empirical investigations show how the GDP rate decreases as corruption increases (Mauro, 1995; Pellegrini \& Gerlagh, 2006). It is also commonly accepted that bribe-maximizing politicians and/or bureaucrats tend to employ resources in those activities that may offer a less transparent management of funds, environmental protection being one of the most prominent examples (see, e.g., Hessami, 2010; Cole, 2006; Lopez \& Mitra, 2000; Pellegrini \& Gerlagh, 2006).

In this paper, we analyse the relationship between public corruption and environmental deterioration. With this aim, we construct an economic setting in which corruption diverts funds allocated for environmental programmes to private pockets through embezzlement and bribery. Rent seeking (embezzlement) by politicians is considered as exogenous in our model. 
Our scientific basis is that corruption can take place only in the presence of interactions between the economic actors. Moreover, such interactions are risky, in that a corrupted transaction may be detected by the authorities. Therefore, the problem is here treated under a game theoretical perspective with incomplete information. The interested reader is referred to Moulin (1986) and Mesterton-Gibbons (2000) for a survey of game theory with applications in economic and social sciences.

An important feature of our model is that corruption—lato sensu-may occur at different levels (see also Wilson \& Damania, 2005).

The players of the game are polluting firms (tax payers), environmental inspectors (tax inspectors) and politicians, and have the option to behave in a corrupt manner. Specifically, on the one hand, a polluting firm attempts to reduce the amount of environmental taxes which it has to pay by bribing a low-level public official (environmental inspector) to make false reports regarding emission levels. In this case, corruption interferes with monitoring, enforcement etc. Hence, more corruption unambiguously corresponds with more pollution. ${ }^{1}$ On the other hand, a high-level public official (a politician) can embezzle part of the environmental revenues, diverting these from environmental policies. The approach we follow is to develop a game with incomplete information. In doing this, we include in the analysis the action of the Nature, which determines randomly the emission level of the polluting firms.

By a purely mathematical perspective, some contributions linking game theory and corruption have appeared in the literature (see, e.g., Macrae, 1982; Pasetta, 1999; Celentani \& Ganuza, 2002; Cerqueti \& Coppier, 2009, 2011, 2013; Cerqueti et al., 2012). However, the aspects related to the interconnections between corruption and environment have been quite neglected. A remarkable exception is Wilson \& Damania (2005), where a theoretical game model is proposed to discuss how corruption affects environmental policies. The main difference between our approach and the quoted paper is that we here want to model the trade-off between different sources of incentives to control corruption, including the ethical sense of the tax payers. Differently, Wilson \& Damania (2005) treat the important problem of the role of political competition in fighting evasion and corruption. Specifically, we assume that when the polluting firm observes that politicians direct the public revenues to the most embezzling activities, it reacts by increasing its evasion rate. On the contrary, if politicians are not corrupt, the polluting firm increases its compliance rates. ${ }^{2}$

In general, the models in literature dealing with States developing strategies to improve fiscal performance have generally focused on the behaviour of the briber. ${ }^{3}$ Suggested policies include augmenting penalties, lowering tax rates and increasing the enforcement of fines. In this respect, we believe that the State may pursue this scope by employing two alternatives strategies: on the one hand, the State can, through greater incentive for the tax inspector, increase the monitoring level (incentive channel). Higher probability of being monitored means for the polluting firm, lesser incentive for evasion and, thus greater tax revenues. Given the public budget constraint, more incentives mean, ceteris paribus, less public resources for the environment (high incentives for inspectors-low environmental expenses); on the other hand, the State can, through greater environmental expenses, increase the compliance of the polluting firm (compliance channel). Higher firm compliance means lesser incentive for evasion and, thus greater tax revenues. Clearly, given the public budget constraint, more environmental expenses

\footnotetext{
1 As stressed in the ESI 2005 report (Esty et al., 2005), "corruption contributes to lax enforcement of environmental regulations and an ability on the part of producers and consumers to evade responsibility for the environmental harms they cause'.

${ }^{2}$ For a more detailed analysis on the efficient environmental tools, i.e. taxes or quotas, see Sandmo (2002).

3 See, for example, Andvig \& Moene (1990).
} 
mean, ceteris paribus, less public resources for tax inspector's incentive (high level of environmental expenses-low incentives) ${ }^{4}$.

As already discussed above, the mathematical model developed here is based on interactions and competition among the economic agents. Therefore, it allows us to capture the tension between such conflicting strategies in pursuing environmental protection, and assess the conditions for which one channel is more effective than the other one.

The paper is organized as follows. In Section 2, we present the model. In Section 3, we describe the timing of the game and provide the main results. In Section 4, we analyse the relationship between environmental revenues and the incentive scheme. Section 5 concludes. All proofs of propositions are in the Appendix.

\section{Theoretical model}

As we said, we consider an economy comprises three players: polluting firms (tax payers), environmental inspectors (tax inspectors) and politicians. Hereafter we will refer to polluting firms as a single representative agent.

We consider a stylized model in which a firm emits pollution that the State attempts to control through an emission tax rate $t \in(0,1)$. We consider only the share of public budget relating to the environmental revenues and environmental expenses. ${ }^{5}$ The State cannot directly observe the level of pollution emitted by the firm and, therefore, employs environmental inspectors to check pollution levels in order to weed out or reduce environmental evasion. The level of emissions is proportional to the firm's production.

Nature decides the production of the polluting firm, and therefore the level of emissions: $y-e$ with probability $p$ and $y$ with probability $1-p$. In the former case, the polluting firm reports the total pollution emission $y-e$, while in the latter case the firm can decide to underreport its emissions by the amount $e$. The environmental evasion can be discovered only if the polluting firm is checked by an environmental inspector. In return for a fixed wage $(\lambda)$, the inspector could ascertain the emission level of the firm and report this information to the State. But, we consider that there is a cost of the effort of inspection $\omega>0$. Therefore, in order to push environmental inspectors to report evasion, the State introduces a bonus rate $\alpha \in[0,1]$ which the environmental inspector obtains on any reported evaded amount.

When the firm declares an emission level $y$, then the environmental inspector does not check it. Otherwise, if an amount of $y-e$ is reported, then the environmental inspector can proceed to the inspection. The inspector who discovers environmental evasion decides whether to report it or to ask for a bribe $\left(b^{d}\right)$ : indeed, it is common knowledge that the environmental inspector is corruptible and open to bribery, in the sense that it pursues its own interest and not necessarily that of the State.

Differently from a wide part of literature which considers that each polluting firm is visited by an environmental inspector, in our model the probability for a polluting firm of being checked is endogenous and different from one. ${ }^{6}$ In fact, the inspector's choice whether to check a polluting firm is based on economic reasoning: the inspector decides whether to check or not depending on the effort-cost of monitoring $\omega$, and on the expected benefit of reporting or not the evasion (the bonus rate $\alpha$ and the bribe, respectively.).

\footnotetext{
${ }^{4}$ See, however, Flatters \& Macleod (1995) and Haque \& Sahay (1996) who consider incentive effects but without explicit consideration of bonus rate, as done here. Our approach is closer to that of Chand \& Moene (1997).

${ }_{6}^{5}$ It is beyond the scope of the present paper to explore the environmental protection policies performed by the State.

${ }^{6}$ See, for example, Mookherjee \& Png (1995) and Damania (2002).
} 
We assume that $\omega \leqslant \alpha e t$, i.e. the environmental inspector's benefit from reporting environmental evasion is higher than the cost of control.

Moreover, we assume that the fixed salary of the environmental inspector is greater than the incentive of discovering corruption, i.e. $\lambda \geqslant \alpha e t$. Therefore, the range of variation of $\alpha$ is in actual fact a restriction of $[0,1]$ and, precisely, $\alpha \in[\underline{\alpha}, \bar{\alpha}]$, where $\underline{\alpha}=\omega /$ et and $\bar{\alpha}=\lambda /$ et .

Since the focus of our research is to analyse the relationship between corruption and environmental quality, we consider only the share of the public budget allocated to the environment. For this reason, in our model, the environmental revenues are used only for environmental protection and emissions abatement activities, along with the payment of environmental inspectors' wages and of the incentives. In this respect, it is necessary to note that, ceteris paribus, the amount of tax revenues allocated for environment protection decreases as the bonus rate $\alpha$ grows.

Furthermore, we assume that politicians - governing the State - are open to corruption and embezzle a percentage $\beta \in[0,1]$ of the environmental revenues. This statement is quite reasonable in that the expenditure in environment protection involves high technology investment on renewable energy and/or abatement, and this leads to expenses that are not always transparent (see Tanzi \& Davoodi, 1997, 2001; Hessami, 2010).

There is an interaction between the State and the polluting firm, in that the polluting firm has the awareness that the under-reported emission is subtracted to the expenses for environmental protection, and this generates a disutility of evading (see Lapatinas et al., 2011; Hessami, 2010). ${ }^{7}$ Simultaneously, the polluting firm knows that a part of the environmental revenues of the State is embezzled by the corrupt politicians, and this reduces the moral incentive not to evade (see Spicer \& Becker, 1980; Andvig \& Moene, 1990; Fortin et al., 2007; Scholz \& Lubell, 1998). In general, from an economic point of view, tax morale should decreases with respect to the political corruption level. Several studies support this assumption. Specifically, Barone \& Mocetti (2011) prove empirically that tax morale increases with an efficient employment of resources, and implicitly state that political corruption leads to less compliance tax payers. Torgler (2003) and Everest-Phillip \& Sandall (2009) show the positive linkage between government's integrity and individuals' tax compliance. Alm \& Gomez (2008) and Akpo (2009) state that government failure in providing good services let citizens be reluctant to pay taxes.

Analogous arguments lead to the decreasing property of tax morale with respect to $\alpha$. Indeed, caeteris paribus, an augment of $\alpha$ means a lower level of environmental protection. Therefore, the perception of the environmental damage due to evasion is reduced. The percentages $\alpha$ and $\beta$ are commonly known by the polluting firm. ${ }^{8}$

To sum up, we introduce a cost-of-evading function associated to the polluting firm ${ }^{9}$

$$
c:[\underline{\alpha}, \bar{\alpha}] \times[0,1]^{2} \times[0,+\infty) \rightarrow[0,1]:(\alpha, \beta, t, e) \mapsto c(\alpha, \beta, t, e),
$$

where $c(\alpha, \beta, t, e)$ is the cost of evasion for the polluting firm, and it represents the firm's perception index of the environmental damage when the bonus rate for the environmental inspector is $\alpha$, the politicians' embezzlement percentage is $\beta$ and the evaded amount is $e t$. The costs $c$ 's disregard on the detection of the environmental evasion, and should be intended as 'moral costs'. By the arguments developed

\footnotetext{
${ }^{7}$ For a more detailed analysis of the relationship between firm's compliance and environmental regulation, see Nyborg \& Telle (2006).

${ }^{8}$ While for $\alpha$ this statement is obvious, a proxy for $\beta$ can be derived by looking at the Corruption Perception Index (CPI), constructed by Transparency International.

9 To the best of our knowledge, this is the first formalization in the literature of tax morale in the environmental corruption context.
} 
above, $c(\alpha, \beta, t, e)$ is assumed to be decreasing with respect to $\alpha$ and $\beta$ and increasing with respect to $t$ and $e$. It is worth stressing that $c(\alpha, \beta, t, e)$ is an economic cost and it does not necessarily have to be measured through units of currency.

In the following, we assume explicitly that

$$
c(\alpha, \beta, t, e)=c(\alpha, \beta) e t .
$$

The definition in formula (1) formalizes that the cost-of-evading term is proportional to the evaded amount with a proportionality factor dependent on $\alpha$ and $\beta$.

We also assume perfect knowledge of the function $c$ by all the actors playing in this game, in the sense that there is an objective measure of the cost-of-evading functions that can be determined by the analysis of the behaviour of the polluting firm.

An explicit shape of function $c$ will be provided only when it will turn out to be useful to describe formally our optimization problems.

When evasion is detected, the environmental inspector can ask the polluting firm for a bribe $b^{d}$. By its perspective, the polluting firm could refuse the bribe or accept to negotiate the amount of $b^{d}$ with the environmental inspector. If the environmental inspector reports the environmental evader, the latter incurs punishment, which is a fine proportional to the evaded amount, where $m \in[0,1]$ is the fine rate, while the former gains a bonus with rate $\alpha$.

Of course, if reported, the firm does not pay the bribe but must pay taxes $t y$, and is affected by the fine met.

\section{The game: description and solution}

Given the framework described above, we can formalize the economic problem into a five-period game with incomplete information.

For a clear exposition, we present the game in a stepwise form. The payoff vector will be indicated with a triple

$$
\underline{\pi}=\left(\pi^{(F)}, \pi^{(S)}, \pi^{(I)}\right),
$$

where $\pi^{(F)}, \pi^{(S)}$ and $\pi^{(I)}$ represent the payoffs of the polluting firm, the State ${ }^{10}$ and the environmental inspector, respectively.

First stage

Nature decides the amount of the firm's emission, that is, $y-e$ with probability $p$ and $y$ with probability $1-p$. The former case will be labelled as good state of Nature, while the latter as bad state of Nature, in accord with the economic sense of low (high) level of emissions, i.e. low (high) level of production. The two cases are analysed separately.

\subsection{Good state of Nature: emissions level $y-e$}

Second stage

The polluting firm declares $y-e$.

\section{Third stage}

\footnotetext{
10 The payoff of the State should be understood as the income from taxes net of the bonus $\alpha$ et eventually paid to the inspector, of the fine met possibly paid by the detected environmental evader and of the wage $\lambda$ of the environmental inspectors.
} 
The inspector must decide whether to check the declared income or not to check it. The game ends in both cases. The payoffs are:

$$
\left\{\begin{array}{lll}
\underline{\pi}_{2}=((1-t)(y-e), t(y-e)-\lambda, \lambda), & & \text { if not checked } \\
\underline{\pi}_{4}=((1-t)(y-e), t(y-e)-\lambda, \lambda-\omega), & & \text { if checked. }
\end{array}\right.
$$

\subsection{Bad state of Nature: emissions level y}

\section{Second stage}

The polluting firm must decide the amount of income to declare: $y$ or $y-e$. If the firm declares $y$, then the environmental inspector does not check the firm and the game ends with the following payoff vector:

$$
\underline{\pi}_{1}=((1-t) y, t y-\lambda, \lambda) .
$$

Otherwise, the game continues to stage 3 .

Third stage

If the polluting firm declares $y-e$, then it pays also the cost-of-evading $c(\alpha, \beta) e t$. The environmental inspector must decide whether to check the emission of the polluting firm or not to check it.

If the environmental inspector does not check the polluting firm, then the game ends with the following payoff vector:

$$
\underline{\pi}_{3}=(y-(y-e) t-c(\alpha, \beta) e t, t(y-e)-\lambda, \lambda) .
$$

Otherwise, the game continues to stage 4 .

Fourth stage

If the environmental inspector checks the polluting firm, then it must decide whether to report the environmental evasion or to ask for a bribe $b^{d}$. If the environmental inspector reports the evasion, then the game ends with the following payoff vector:

$$
\underline{\pi}_{5}=((1-t) y-m e t, t y+(m-\alpha) e t-\lambda, \lambda-\omega+\alpha e t) .
$$

Otherwise, the game continues to stage 5.

Fifth stage

If the environmental inspector asks the polluting firm for a bribe $b^{d}>0$, then the firm must decide whether to negotiate such a bribe or refuse the negotiation. When the bribe is refused by the firm, then the game ends with the following payoff vector:

$$
\underline{\pi}_{6}=((1-t) y-m e t, t y+(m-\alpha) e t-\lambda, \lambda-\omega+\alpha e t) .
$$

If the polluting firm accepts to negotiate the bribe, then the negotiation starts and the two parties will find an agreement on the bribe $b^{N B}$, which corresponds to the Nash solution to a bargaining game. The polluting firm pays the bribe and it is not reported, and the game ends with the payoff vector given by

$$
\underline{\pi}_{7}=\left(y-(y-e) t-b^{N B}-c(\alpha, \beta) e t, t(y-e)-\lambda, \lambda-\omega+b^{N B}\right) .
$$

We provide an explicit expression of the bribe $b^{N B}$.

Proposition 3.1 There is a unique bribe $b^{N B}$, as the Nash solution to the bargaining game, given by

$$
b^{N B}=\frac{1}{2} \cdot[1-c(\alpha, \beta)+\alpha+m] e t .
$$


We now formalize the solution of the game, which has been derived by using the backward induction method starting from the last stage.

Proposition 3.2 There exist four numbers $p_{1}, p_{2} \in[0,1]$ and $M_{1}, M_{2} \in[0,+\infty)$, defined as in (A.8), (A.21), (A.2) and (A.6), respectively, such that the following cases hold.

(I) Assume that $c(\alpha, \beta)+\alpha>1$.

(I-a) If $1-p \leqslant p_{1}$, then

(I-a.1) if $m \leqslant M_{2}$, the game ends with random payoff vector

$$
\underline{\pi}_{A}= \begin{cases}\underline{\pi}_{3} & \text { with probability } 1-p, \\ \underline{\pi}_{2} & \text { with probability } p ;\end{cases}
$$

(I-a.2) if $m>M_{2}$, the game ends with random payoff vector

$$
\underline{\pi}_{B}= \begin{cases}\underline{\pi}_{1} & \text { with probability } 1-p, \\ \underline{\pi}_{4} & \text { with probability } p .\end{cases}
$$

(I-b) If $1-p>p_{1}$, then the game ends with random payoff vector $\underline{\pi}_{B}$.

(II) Assume that $c(\alpha, \beta)+\alpha \leqslant 1$.

(II-a) If $1-p \leqslant p_{2}$, then:

(II-a.1) if $m \leqslant M_{2}$, the game ends with random payoff vector $\underline{\pi}_{A}$;

(II-a.2) if $m>M_{2}$, the game ends with random payoff vector $\underline{\pi}_{B}$.

(II-b) If $1-p>p_{2}$, then:

(II-b.1) if $m \leqslant M_{2}$, the game ends with random payoff vector $\underline{\pi}_{A}$;

(II-b.2) if $M_{2}<m \leqslant-M_{1}$, the game ends with random payoff vector

$$
\underline{\pi}_{C}= \begin{cases}\underline{\pi}_{7} & \text { with probability } 1-p, \\ \underline{\pi}_{4} & \text { with probability } p .\end{cases}
$$

(II-b.3) if $m>-M_{1}$, the game ends with random payoff vector $\underline{\pi}_{B}$.

It is worth noting that the thresholds $M_{1}$ and $M_{2}$, defined, respectively, in (A.2) and (A.6), depend on the parameters $\alpha$ and $\beta$. Substantially, the fine rate thresholds are adequate to the levels $\beta$ of the corrupt politicians and $\alpha$ of the environmental revenues devoted to the payment of the bonus to the environmental inspector. In the formulas we should correctly write

$$
M_{1} \equiv M_{1}(\alpha, \beta), \quad M_{2} \equiv M_{2}(\alpha, \beta) .
$$

Analogously, we should write

$$
p_{1} \equiv p_{1}(\alpha), \quad p_{2} \equiv p_{2}(\alpha, \beta)
$$


It is not necessary to report in this section the explicit reference to $\alpha$ and $\beta$ of the $p$ 's and the $M$ 's, which will be reintroduced in the section devoted to the analysis of the environmental revenues.

Furthermore, Proposition 3.2 highlights also that the game involves the active interaction between the polluting firm and environmental inspectors. Such an interaction leads to different perfect Bayesian equilibria, which depend on the values assumed by the parameters. The main distinction to perform is the one associated to the aggregation of the cost-of-evading $c(\alpha, \beta)$ and bonus rate $\alpha$. Such a distinction is based on the aggregate strength of the 'enemies of evasion', which is high in the case of $c(\alpha, \beta)+\alpha>$ 1 and low otherwise. A further distinction is that concerning the occurrence of the good or bad state of Nature and that related to the amount of the fine rate $m$.

If the emission of the polluting firm is $y-e$, two equilibria without environmental evasion occur:

- $\underline{\pi}_{2}$ is associated to the equilibrium with no check;

- $\underline{\pi}_{4}$ is associated to the equilibrium with check.

When the emission of the polluting firm is $y$, then three equilibria occur:

- $\underline{\pi}_{1}$ is associated to the equilibrium with no environmental evasion;

- $\underline{\pi}_{3}$ is associated to the equilibrium with undetected environmental evasion;

- $\underline{\pi}_{7}$ is associated to the equilibrium with environmental evasion and fiscal corruption.

To provide a comment on the results listed in Proposition 3.2, it is worth focusing only on the case of occurrence of the bad state of Nature. For the sake of clarity, we repeat the distinction used in Proposition 3.2 .

(I) Suppose that the value of the aggregation of the cost-of-evading function and the bonus rate is high. In this case the evasion is strongly contrasted by the aggregation of the incentive schemes for the inspectors and of the costs sustained by the evaders.

(I-a) Suppose that the occurrence of the bad state of Nature is rather improbable.

(I-a.1) If the fine rate is small, then the polluting firm is not scared about the punishment. Moreover, the bribe is small as well, in that it is proportional to the fine rate. Hence, the polluting firm underreports its emission. The environmental inspector does not check the polluting firm, basically for two reasons: in the one end, since the fine rate is small and the bribe goes hand in hand with the fine rate, the eventual bribe would also be small; on the other, the probability that the emission $y-e$ effectively gained by the polluting firm is so high that there is a concrete risk of a nonuseful effort in checking.

(I-a.2) If the fine rate is high, then the polluting firm is worried about the punishment or the amount of the bribe and finds it worthwhile to report its emission. The environmental inspector checks the polluting firm, because the fine rate is so high that the bribe is great enough to incentive the effort of checking.

(I-b) Suppose that the probability of the occurrence of the bad state of Nature is high.

In this case, the bad state of Nature is so probable that the inspector finds it worthwhile to check the emission of the polluting firm and the firm finds it worthwhile to avoid the probable environmental inspector's actions by reporting its emission. This behaviour is 
supported also by the high value of the sum between $\alpha$ and $c(\alpha, \beta)$, which means that there is a discouragement for the polluting firm to evade and an encouragement for the inspector to check.

(II) Now, suppose that the value of the aggregation of the cost-of-evading function and the bonus rate is low. Evasion is facilitated by the low level of the aggregation of the incentive schemes for the inspectors and of the costs sustained by the evaders.

(II-a) Suppose that there is a high probability of the occurrence of the good state of Nature.

(II-a.1) If the fine rate is small, then the polluting firm finds it worthwhile to evade taxes and, if detected, to agree on the small bribe. The bribe is so small that the environmental inspector does not find it worthy to inspect the polluting firm.

(II-a.2) If the fine rate is high, then the punishment and the bribe are too high and the polluting firm finds it worthwhile to report its emission. The environmental inspector checks the polluting firm, for the same reasons listed in case (I-a.2), which are amplified in this case.

(II-b) Suppose that the probability of the occurrence of the bad state of Nature is quite high.

(II-b.1) If the fine rate is small, then the same arguments of case (II-a.1) apply, and we are in the situation of no checked underreported income.

(II-b.2) If the fine rate is medium, then the bribe is still low enough to convince the polluting firm to underreport its emission. By the perspective of the environmental inspector, the bribe is so high that it is worthwhile to check the emission of the polluting firm.

(II-b.3) If the fine rate is high, then the polluting firm finds it worthwhile to report its emission because of the high amounts of fine and bribe and due to the high probability to be checked by the environmental inspector. The bribe is so high that the environmental inspector finds it worthwhile to check the polluting firm, and this confirms the case (II-b.2).

\section{Optimization of the expected environmental revenues}

In this section we focus on the expected environmental revenues of the State. The randomness of the environmental revenues is grounded on the evidence that the polluting firm may experience an emission level $y$ with probability $1-p$ or $y-e$ with probability $p$.

Proposition 3.2 implicitly states three important facts regarding the income of the State.

Firstly, the environmental revenues of the State depends on the value of the aggregation of cost-ofevading function and bonus rate. This is the formalization of the existence of a relationship between the strength of the enemies of evasion and the environmental revenues.

Secondly, the expected aggregate environmental revenues depend on the probability of occurrence of the bad state of Nature.

Thirdly, the aggregate environmental revenues vary according to the amount of the fine paid by the reported polluting firm. In this respect, it is here necessary to highlight the dependence of the thresholds 
$M_{1}$ and $M_{2}$, defined, respectively, in (A.2), and (A.6), on $\alpha$ and $\beta$. Hence, $M_{1}$ and $M_{2}$ will be interpreted hereafter as in (10). Analogously, $p_{1}$ and $p_{2}$ will be read as in (11).

As already pre-announced above, in order to analyse the environmental expected revenues of the State, it now turns out to be useful to provide an explicit expression for the cost-of-evading function $c(\alpha, \beta)$. A suitable choice is to assume

$$
c(\alpha, \beta)=\exp \{-\alpha(\beta+1)\} .
$$

Under definition (12), the function $c(\alpha, \beta)$ decreases with respect to $\alpha$ and $\beta$ and $c(\alpha, \beta) \in[0,1]$, for each $\alpha \in[\underline{\alpha}, \bar{\alpha}]$ and $\beta \in[0,1]$. We proceed hereafter by replacing $c(\alpha, \beta)$ with its expression in formula (12).

From (12), the critical condition of Proposition 3.2, namely $c(\alpha, \beta)+\alpha>1$, can be rewritten in terms of an inequality involving $\alpha$ and $\beta$ as follows:

$$
\beta<\frac{-\log (1-\alpha)}{\alpha}-1
$$

REMARK 4.1 Evidently, the term $-\log (1-\alpha) / \alpha-1$ in (13) does not belong always to [0,1]. As an example, $-\log (1-\alpha) / \alpha-1>1$ as $\alpha$ approaches 1 . In this particular case, condition in (13) is trivially satisfied. Differently, if $\alpha$ is close to zero, a Taylor expansion leads to

$$
-\frac{\log (1-\alpha)}{\alpha}-1 \sim \frac{-\alpha-\alpha^{2}}{-\alpha}-1=\alpha \in(0,1) .
$$

In this case (13) is fulfilled only by some values of $\beta$. In all the cases, the analysis carried out throughout the paper maintains its validity.

Now, denote by $r(\alpha, \beta)$ the contribution given by the representative polluting firm to the environmental revenues. Proposition 3.2 leads straightforwardly to an explicit expression for $r(\alpha, \beta)$ :

Proposition 4.1 Consider $(\alpha, \beta) \in[\underline{\alpha}, \bar{\alpha}] \times[0,1]$ and introduce the following subset of $[0,1]^{2}$ :

$$
\mathcal{A}(\alpha, \beta)= \begin{cases}\mathcal{A}_{1}(\alpha, \beta), & \text { if (13) holds; } \\ \mathcal{A}_{2}(\alpha, \beta), & \text { if (13) does not hold, }\end{cases}
$$

where

$$
\begin{aligned}
\mathcal{A}_{1}(\alpha, \beta)= & \left\{\left[1-\frac{\omega}{\alpha e t}, 1\right] \times\left(\frac{2 \omega}{e t(1-p)}+\exp \{-\alpha(\beta+1)\}-\alpha-1,1\right]\right. \\
& \left.\cup\left[0,1-\frac{\omega}{\alpha e t}\right] \times[0,1]\right\} \cap[0,1]^{2}
\end{aligned}
$$

and

$$
\begin{aligned}
\mathcal{A}_{2}(\alpha, \beta)= & \left\{\left[1-\frac{\omega}{\operatorname{et}[1-\exp \{-\alpha(\beta+1)\}]}, 1\right] \times\left(\frac{2 \omega}{\operatorname{et}(1-p)}+\exp \{-\alpha(\beta+1)\}-\alpha-1,1\right]\right. \\
& \left.\cup\left[0,1-\frac{\omega}{\operatorname{et}[1-\exp \{-\alpha(\beta+1)\}]}\right] \times(1-\exp \{-\alpha(\beta+1)\}-\alpha, 1]\right\} \cap[0,1]^{2} .
\end{aligned}
$$


Then

$$
r(\alpha, \beta)= \begin{cases}(1-\beta) t y, & \text { if }(p, m) \in \mathcal{A}(\alpha, \beta) ; \\ (1-\beta) t(y-e), & \text { if }(p, m) \notin \mathcal{A}(\alpha, \beta),\end{cases}
$$

The set $\mathcal{A}(\alpha, \beta)$ may be interpreted as the region where no evasion occurs.

The expected value of the contribution of the representative environmental payer when $\alpha$ and $\beta$ are fixed is

$$
\pi(\alpha, \beta)=p(1-\beta) t(y-e)+(1-p) r(\alpha, \beta),
$$

where $r(\alpha, \beta)$ is defined as in (17).

\subsection{The optimization problem}

We consider the case in which the State aims at maximizing the total expected environmental revenues. To this aim, each contribution $\pi(\alpha, \beta)$ in (18) should be maximized with respect to $\alpha$. The percentage of embezzlement $\beta$ is assumed to be maintained by the corrupt Government, and it represents an exogenous parameter of the model.

It is important to note that $\alpha$ produces two contrasting effects in the fight against environmental evasion:

1 Positive effect. On the one hand, a higher level of $\alpha$ increases the incentive for the environmental inspector to control environmental evasion. This happens because a higher level of $\alpha$ means a higher bonus if the inspector denounces the detected evasion, but at the same time, it means a greater bribe for the inspector who does not report the detected evasion. So, through this effect, a higher level of $\alpha$ means less incentive for environmental evasion and, ceteris paribus, more environmental revenues.

2 Negative Effect. On the other hand, a higher level of $\alpha$ means, ceteris paribus, fewer resources devoted to the environment and therefore a lower cost of evasion (c) for the polluting firm. So, a higher level of $\alpha$ implies, trough this channel, a higher evasion and, ceteris paribus, lower environmental revenues.

The optimization problem can be formalized as follows.

4.1.1 Problem $\mathbf{P} 1$ Fix $\beta \in[0,1]$. We search for the optimal bonus rate $\alpha_{\beta}^{\star}$ such that:

$$
\pi\left(\alpha_{\beta}^{\star}, \beta\right)=\sup _{\alpha \in[\underline{\alpha}, \bar{\alpha}]} \pi(\alpha, \beta)
$$

By formula (18), Problem $\mathbf{P} 1$ is equivalent to the following:

4.1.2 Problem $\mathbf{P} 1^{\prime} \quad$ Fix $\beta \in[0,1]$. We search for the optimal bonus rate $\alpha_{\beta}^{\star}$ such that:

$$
\left|\mathcal{A}\left(\alpha_{\beta}^{\star}, \beta\right)\right|=\sup _{\alpha \in[\underline{\alpha}, \bar{\alpha}]}|\mathcal{A}(\alpha, \beta)|,
$$

where $|\bullet|$ denotes the measure of the bi-dimensional set $\bullet$. 
A closed-form theoretical result for solving Problem $\mathbf{P} 1^{\prime}$ is contained in the following statement:

Proposition 4.2 Assume that $\underline{\alpha}>(1-p) / 2$. Then both $\left|\mathcal{A}_{1}(\alpha, \beta)\right|$ and $\left|\mathcal{A}_{2}(\alpha, \beta)\right|$ increase with respect to $\alpha$, for each $\beta \in[0,1]$.

Proposition 4.2 offers some insights on the size of the honesty region, and hence on the environmental revenues. If the lower level of the bonus rate is high enough, then the State should incentivize the monitoring activity to efficiently fight evasion. The meaning of this outcome lies in the definition of the cost function, which decreases with respect to $\alpha$. Indeed, if the monitoring activity is rather high, then the public expenditure for the environment is so low that pollutant firms do not judge convenient to pay taxes for environmental protection and evade. The unique way to reduce evasion is then to implement a more stringent monitoring activity by incrementing $\alpha$. The threshold $(1-p) / 2$ grows with the probability of the occurrence of the bad state of Nature. Basically, the pollutant firms seem to be more tolerant to a reduction of funds for the environmental protection when the pollution emission is high. In this case, therefore, the former (positive) effect of $\alpha$ prevails on the negative one. In fact, as $\alpha$ grows the greater level of control pushes the polluting firms to evade less, and therefore, the State will have greater environmental revenues.

Unfortunately, a closed form result is not available for the case $\underline{\alpha} \leqslant(1-p) / 2$. Moreover, in the context we are dealing with, simulations appear to us non exhaustive, being the parameter set involved in our theoretical model too wide. However, we can proceed by simulations with the specific aim to test if the result of Proposition 4.2 can be reverted when $\underline{\alpha}$ is small.

The used dataset consists in: $\omega=1, \lambda=2, e=1000, t=0.4$. These values have a clear justification in standard economic theory and in important references. The effort of inspecting $\omega$ can be interpreted as the marginal disutility of labour, which must be reasonably smaller than the wage. In the numerical experiments, we then state the theoretical hypothesis of a wage doubling the disutility of labour. The amount of evasion of the firm can be taken several times larger than the wage of a single individual, and this explains the choice of $e=1000$. The only parameter which should be better contextualized in the literature is, to our opinion, the tax rate $t$. In this respect, it is worth pointing out that the value $t=0.4$ has been adopted by several important environmental researches (see, for example, Goulder et al., 1999; Parry et al., 1999; West \& Williams, 2004).

The probability $p$ is assumed to vary in a range [0,0.99]. This parameter set implies that $\underline{\alpha}<$ $(1-p) / 2$. We consider a country with a fair level of political corruption, and assume $\beta=0.5$. The plot of the surface $z=|\mathcal{A}(\alpha, \beta)|$ drawn with respect to $\alpha$ and $p$ is reported in Fig. 1 .

Figure 1 provides some interesting suggestions. We note that, once $p$ has been fixed, the surface $|\mathcal{A}(\alpha, \beta)|$ decreases with respect to $\alpha$. This evidence meets our basic requirement, which was to test if the result of Proposition 4.2 may be reverted for $\underline{\alpha} \leqslant(1-p) / 2$. Hence, a lower level of monitoring activity rather small may lead to the strategy for which the State should increment the expenses for environmental protection to achieve a greater level of expected environmental revenues. This outcome is due to the costs that pollutant firms pay in evading, which are particularly relevant when the level of environmental funds are high. In this case, when the minimum level of $\alpha$ is low enough, i.e. $\underline{\alpha} \leqslant$ $(1-p) / 2$, the negative effect of $\alpha$ prevails on the positive one. As $\alpha$ increases, the evasion increases too and, therefore, the environmental revenues decrease. Thus, the State must fix a low level of bonus rate $\alpha$, in order to maximize the environmental revenues.

To sum up, two perspectives can be compared. On the one hand, a policy-maker who fixes a small maximum level to the expenses for environmental protection (case $\underline{\alpha}>(1-p) / 2$ ) must be aware that the only way to fight evasion is to implement a growing level of monitoring activity (hence, reducing the funds for the environment). On the other hand, a large maximum level of expenses for environmental 


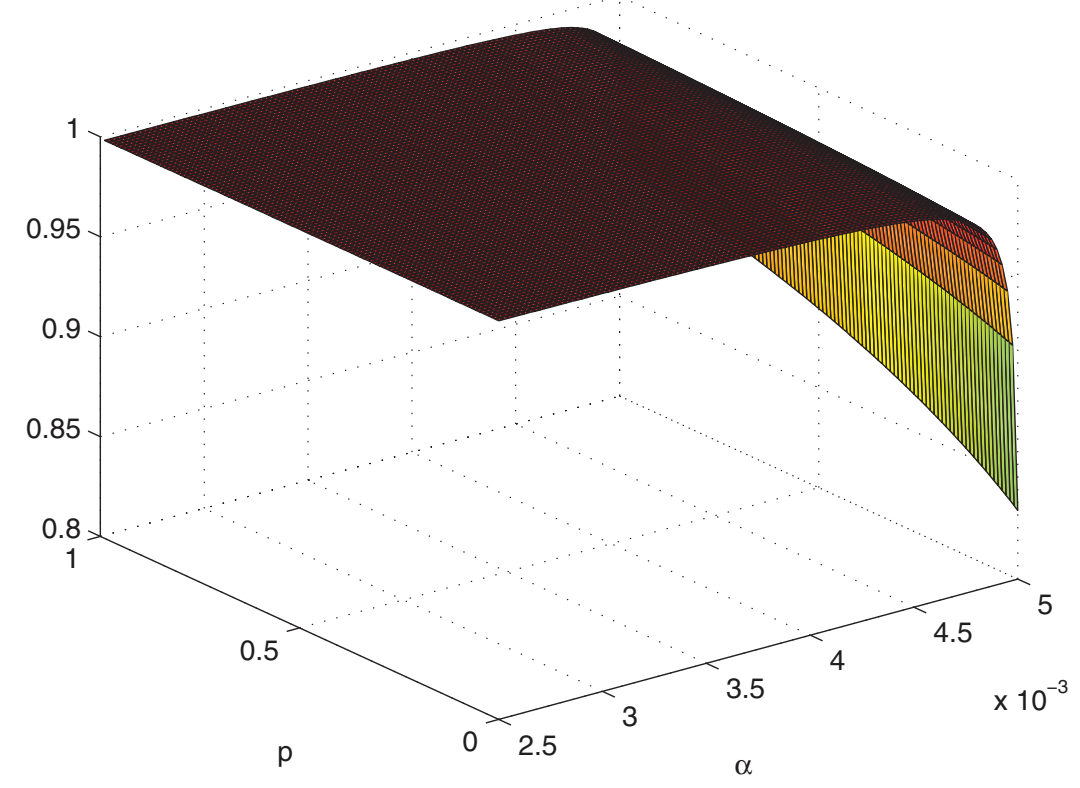

FIG. 1. The surface $z=|\mathcal{A}(\alpha, \beta)|$ drawn with respect to $\alpha$ and $p$, for $\beta=0.5$.

protection may lead to a more ethical route-based on the cost-of-evading functions of the pollutant firms - to fight evasion, which drives the policy-maker towards an improvement of the environmental expenses.

\section{Conclusions}

In our paper, we develop and solve a theoretical game model with incomplete information to examine the interaction between polluting firms, environmental inspectors and politicians. In our model, each of these players have the option to behave in a corrupt manner and their interaction has an effect on environmental protection. To be more precise, on the one hand, a polluting firm attempts to reduce the amount of environmental taxes which it has to pay by bribing a low level public official (environmental inspector) to make false reports regarding emission levels; on the other hand, a high-level public official (a politician) can embezzle part of the environmental revenues, diverting these from environmental policies. We consider the case in which the State aims at maximizing the total expected environmental revenues. In doing this, the State employs two conflicting strategies based on punishment (high incentives for inspectors and, consequently, low level of environmental expenses) or on polluting firms compliance (high level of environmental expenses and, consequently, low incentives). We find that the effect of bonus rate in reducing evasion is ambiguous. In fact, if the State fixes a high minimum incentive level, the only way to fight evasion is to implement a growing level of monitoring activity (hence, reducing the funds for the environment). Conversely, a low minimum incentive threshold for the environmental inspector may lead to a more ethical route-based on the cost-of-evading functions of the pollutant firms - to fight evasion, which drives the policy-maker towards an improvement of the fiscal performance. 


\section{REFERENCES}

AкPо, U. (2009) The people as government: the importance of tax payment. Akwa Ibom State Revenue Summit. Uyo: Akwa Ibom State Internal Revenue Service.

Alm, J. \& Gomez, J. L. (2008) Social capital and tax morale in Spain. Eco. Anal. Pol., 38, 34-47.

Andvig, J. C. \& Moene, K. O. (1990) How corruption may corrupt. J. Econ. Behav. Organ., 13, 63-76.

Barone, G. \& Mocetti, S. (2011) Tax morale and public spending inefficiency. Int. Tax Public Finance, 18, 724749.

Bosetti, V., Messina, E. \& Valente, P. (2002) Optimization technologies and environmental applications. IMA J. Manag. Math., 13, 167-185.

Celentani, M. \& Ganuza, J.-J. (2002) Organized vs. competitive corruption. Ann. Oper. Res., 109, 293-315.

Cerqueti, R. (2013) Exhaustion of resources: a marked temporal process framework. Stoch. Environ. Res. Risk Assess. Doi:10.1007/s00477-013-0798-7.

Cerqueti, R. \& Coppier, R. (2009) Tax revenues, fiscal corruption and 'shame' costs. Econ. Model., 26, 12391244.

Cerqueti, R. \& Coppier, R. (2011) Economic growth, corruption and tax evasion. Econ. Model., 28, 489-500.

Cerqueti, R. \& Coppier, R. (2013) Corruptibility and tax evasion. Eur. J. Law Econ. Doi:10.1007/s10657-0139406-z.

Cerqueti, R., Coppier, R. \& Piga, G. (2012) Corruption, growth and ethnolinguistic fractionalization: a theoretical game model. J. Econ., 106, 153-181.

Chand, S. \& Moene, K. O. (1997) Controlling fiscal corruption. IMF Working Paper, no. 97/10, 1-17.

Cole, M. A. (2006) Does trade liberalization increase national energy use?. Econ. Lett., 92, 108-112.

DAmania, R. (2002) Environmental controls with corrupt bureaucrats. Environ. Dev. Econ., 7, 407-427.

Esty, D. C., Marc Levy, A., Srebotnjak, T. \& De Sherbinin, A. (2005) Environmental Sustainability Index: Benchmarking National Environmental Stewardship. New Haven, CT, USA: Yale Center for Environmental Law and Policy.

Everest-Phillip, M. \& SANDALl, R. (2009) Linking business tax reform with governance: how to measure success. Working paper. Investment Climate Department, World Bank Group.

Flatters, F. \& Macleod, W. (1995) Administrative corruption and taxation. Int. Tax Public Finance, 2, 397-417.

Fortin, B., Lacroix, G. \& Villeval, M. C. (2007) Tax evasion and social interactions. J. Public Econ., 91, 20892112.

Goulder, L. H., Parry, I. W. H., Williams III, R. C. \& Burtraw, D. (1999) The cost-effectiveness of alternative instruments for environmental protection in a second-best setting. J. Public Econ., 72, 329-360.

Haque, N. U. \& SAHAY, R. (1996) Do government wage cuts close budget deficits? A conceptual framework for developing countries and transition economies. IMF Working Papers 96/19. International Monetary Fund.

Hessami, Z. (2010) Corruption and the composition of public expenditures: evidence from OECD countries. MPRA Paper 25945.

Lapatinas, A., Litina, A. \& Sartzetakis, E. (2011) Corruption and environmental policy: an alternative perspective. Discussion Paper No. 08/2011.

Lopez, R. \& Mitra, S. (2000) Corruption, pollution, and the Kuznets environment curve. J. Environ. Econ. Manage., 40, 137-150.

MACRAE, J. (1982) Underdevelopment and the economics of corruption: a game theory approach. World Dev., 10, $677-687$.

Mauro, P. (1995) Corruption and growth. Quart. J. Econ., 110, 681-712.

Mesterton-Gibbons, M. (2000) An Introduction to Game-theoretic Modelling. Rhode Island: American Mathematical Society.

MookherJee, D. \& PNG, I. P. L. (1995) Corruptible law enforcers: how should they be compensated. Econ. J., 105, $145-159$.

Moulin, H. (1986) Game Theory for the Social Sciences, 2nd and revised edn. New York: New York University Press. 
Nyborg, K. \& Telle, K. (2006) Firm's compliance to environmental regulation: is there really a paradox? Environ. Resource Econ., 35, 1-18.

Parry, I. W. H., Williams III, R. C. \& Goulder, L. H. (1999) When can carbon abatement policies increase welfare? The fundamental role of distorted factor markets. J. Environ. Econ. Manage., 37, 52-84.

Pasetta, V. (1999) Dynamics in divide the money game with bribing. Ann. Oper. Res., 88, 361-377.

Pellegrini, L. \& Gerlagh, R. (2006) Corruption, democracy, and environmental policy: an empirical contribution to the debate. J. Environ. Dev., 15, 332-354.

SANDmo, A. (2002) Efficient environmental policy with imperfect compliance'. Environ. Resource Econ., 23, 85103.

Scholz, J. T. \& Lubell, M. (1998) Trust and taxpayers: testing the heuristic approach to collective action. Am. J. Polit. Sci., 42, 398-417.

Spicer, M. \& Becker, L. A. (1980) Fiscal inequity and tax evasion: an experimental approach. Nat. Tax J., 33, $171-175$.

TanZI, V. \& Davoodi, H. (1997) Corruption, public investment, and growth', IMF Working Paper, no. 97/139.

Tanzi, V. \& Davoodi, H. (2001) Corruption, Growth and Public Finances (A. J. Kein ed.), Political Economy of Corruption, London, Routledge, 89-110.

TAPIERo, C. S. (2009) Energy consumption and environmental pollution: a stochastic model. IMA J. Manage. Math., 20, 263-273.

Torgler, B. (2003) Tax morale: theory and analysis of tax compliance. Unpublished doctoral dissertation, University of Zurich, Switzerland.

West, S. E. \& WiLliams III, R. C. (2004) Empirical estimates for environmental policy making in a second-best setting. National Bureau of Economic Research, Inc, NBER Working Papers 01/2004; DOI:10.2139/ssrn.504202.

Wilson, J. K. \& DAMANiA, R. (2005) Corruption, political competition and environmental policy. J. Environ. Econ. Manage., 49, 516-535.

\section{Appendix}

\section{A.1 Proof of Proposition 3.1}

Let $\underline{\phi}_{\Delta}=\left(\phi_{\Delta}^{(E)}, \phi_{\Delta}^{(I)}\right)$ be the vector of the differences in the payoffs between the case of agreement and disagreement regarding the bribe between the polluting firm and the environmental inspector, that is

$$
\phi_{\Delta}^{(E)}=\pi_{7}^{(E)}-\pi_{6}^{(E)}, \quad \phi_{\Delta}^{(I)}=\pi_{7}^{(I)}-\pi_{6}^{(I)} .
$$

We assume that polluting firm and environmental inspector have equal power in bargaining. Hence, follow the generalized Nash bargaining theory, the bribe of agreement comes out from $\max _{b \in[0,+\infty)} \phi_{\Delta}^{(E)}$. $\phi_{\Delta}^{(I)}$, that is

$$
\max _{b \in[0,+\infty)}[\operatorname{et}(1-c(\alpha, \beta)+m)-b] \cdot[b-\alpha e t] .
$$

The objective function in (A.1) is a reversed U-shaped quadratic function in $b$. Therefore, the first-order condition leads to the bribe of agreement $b^{N B}$ as in (9), which is the unique equilibrium bribe in the last sub-game.

\section{A.2 Proof of Proposition 3.2}

The static game is solved using backward induction, which enables the equilibria to be obtained.

(5) At stage 5, the polluting firm negotiates the bribe if and only if $\pi_{7}^{(E)}-\pi_{6}^{(E)}>0$, which is verified when the amount of the fine satisfies the following inequality:

$$
m>M_{1} \equiv c(\alpha, \beta)+\alpha-1
$$


(4) Ascending the decision-making tree, at stage 4 the environmental inspector decides whether to ask for a bribe or not.

(4.1) if $m>M_{1}$ is verified, then the bribe is requested by the environmental inspector if and only if $\pi_{7}^{(I)}-\pi_{5}^{(I)}>0$, which is always true.

(4.2) if $m \leqslant M_{1}$, then environmental inspector's payoffs are equivalent in both of cases of requested or not requested bribe, and so she/he will avoid to ask for the bribe.

(3) At stage 3, polluting firm declares $y-e$ and environmental inspector must decide whether to inspect her/his income or not. This decision is driven by the expected payoffs, in that the environmental inspector does not know whether the income of the polluting firm is $y$ or $y-e$. Denote as $\pi_{C}^{(I)}$ and $\pi_{N C}^{(I)}$ the random variables associated to the payoff of the environmental inspector when checking or not checking the polluting firm, respectively.

Environmental inspector checks the polluting firm if and only if

$$
\mathbf{E}\left[\pi_{C}^{(I)}\right]-\mathbf{E}\left[\pi_{N C}^{(I)}\right]>0,
$$

where $\mathbf{E}$ is the expected value operator and

$$
\mathbf{E}\left[\pi_{N C}^{(I)}\right]=p \pi_{2}^{(I)}+(1-p) \pi_{3}^{(I)}=\lambda .
$$

(3.1) If $m>M_{1}$, then

$$
\begin{aligned}
\mathbf{E}\left[\pi_{C}^{(I)}\right] & =p \pi_{4}^{(I)}+(1-p) \pi_{7}^{(I)} \\
& =p(\lambda-\omega)+(1-p)\left[\lambda-\omega+\frac{1}{2} \cdot(1-c(\alpha, \beta)+\alpha+m) e t\right] .
\end{aligned}
$$

Therefore, condition (A.3) is equivalent to

$$
m>M_{2} \equiv \frac{1}{e t} \cdot \frac{2 \omega}{1-p}+c(\alpha, \beta)-\alpha-1 .
$$

(3.2) If $m \leqslant M_{1}$, then

$$
\mathbf{E}\left[\pi_{C}^{(I)}\right]=p \pi_{4}^{(I)}+(1-p) \pi_{5}^{(I)}=p(\lambda-\omega)+(1-p)(\lambda-\omega+\alpha e t) .
$$

Therefore, condition (A.3) is equivalent to

$$
1-p>p_{1} \equiv \frac{\omega}{\alpha e t} .
$$

(2) At stage two the polluting firm must decide whether to underreport her/his income.

The case with production $y-e$ is trivial, while if the income is $y$, then the decision is driven by the payoffs.

Now, suppose that the income of the polluting firm is $y$, i.e. the bad state of the Nature occurs. Denote as $\pi_{R}^{(E)}$ and $\pi_{N R}^{(E)}$ the payoff of the polluting firm when reporting $y$ or $y-e$, respectively. 
Pollution firm reports her/his income if and only if

$$
\pi_{R}^{(E)}-\pi_{N R}^{(E)}>0
$$

where

$$
\pi_{R}^{(E)}=\pi_{1}^{(E)}=(1-t) y .
$$

For evaluating $\pi_{N R}^{(E)}$, we need to distinguish some cases.

(2.1) If one of the following two cases occurs:

$$
\left\{\begin{array}{l}
M_{1}<m \leqslant M_{2} ; \\
m \leqslant M_{1} \text { and } 1-p \leqslant p_{1},
\end{array}\right.
$$

then:

$$
\pi_{N R}^{(E)}=\pi_{3}^{(E)}=y-t(y-e)-c(\alpha, \beta) e t
$$

By (A.10) and (A.12), condition (A.9) becomes

$$
e t[c(\alpha, \beta)-1]>0 \text {, }
$$

that is never verified, being $c(\alpha, \beta) \in[0,1]$. The game ends with random payoff

$$
\begin{cases}\underline{\pi}_{3} & \text { with probability } 1-p \\ \underline{\pi}_{2} & \text { with probability } p .\end{cases}
$$

(2.2) If $m>\max \left\{M_{1}, M_{2}\right\}$, then

$$
\pi_{N R}^{(E)}=\pi_{7}^{(E)}=y-(y-e) t-c(\alpha, \beta) e t-\frac{1}{2} \cdot[1-c(\alpha, \beta)+\alpha+m] e t .
$$

By (A.10) and (A.14), we can rewrite condition (A.9) as follows:

$$
m>-M_{1} \equiv 1-c(\alpha, \beta)-\alpha .
$$

If (A.15) is true, then the game ends with random payoff

$$
\begin{cases}\underline{\pi}_{1} & \text { with probability } 1-p \\ \underline{\pi}_{4} & \text { with probability } p\end{cases}
$$

If (A.15) is not satisfied, then the game ends with random payoff

$$
\begin{cases}\underline{\pi}_{7} & \text { with probability } 1-p \\ \underline{\pi}_{4} & \text { with probability } p .\end{cases}
$$


(2.3) If $m \leqslant M_{1}$ and $1-p>p_{1}$, then

$$
\pi_{N R}^{(E)}=\pi_{5}^{(E)}=y(1-t)-\text { met. }
$$

By (A.10) and (A.18), condition (A.9) is trivially verified, as expected. The game ends with random payoff

$$
\begin{cases}\underline{\pi}_{1} & \text { with probability } 1-p \\ \underline{\pi}_{4} & \text { with probability } p .\end{cases}
$$

To complete the proof, it is sufficient to apply some simple algebra and notice that:

$$
\begin{cases}M_{1}>M_{2} & \text { if and only if } 1-p>p_{1} \\ M_{1}>0 & \text { if and only if } c(\alpha, \beta)+\alpha>1 \\ -M_{1}>M_{2} & \text { if and only if } 1-p>p_{2}\end{cases}
$$

where

$$
p_{2}=\frac{\omega}{e t(1-c)}
$$

\section{A.3 Proof of Proposition 4.2}

(i) By formula (15) we have

$$
\left|\mathcal{A}_{1}(\alpha, \beta)\right|=\frac{\omega}{\alpha e t} \cdot\left[1+\alpha-\exp \{-\alpha(\beta+1)\}-\frac{2 \omega}{e t(1-p)}\right]+1
$$

Hence

$$
\begin{aligned}
\frac{\partial\left|\mathcal{A}_{1}(\alpha, \beta)\right|}{\partial \alpha} & =\frac{\omega}{\alpha e t} \cdot\left\{(\beta+1) \exp \{-\alpha(\beta+1)\}+\frac{1}{\alpha}\left[\exp \{-\alpha(\beta+1)\}+\frac{2 \omega}{\operatorname{et}(1-p)}-1\right]\right\} \\
& >\frac{\omega}{\alpha e t} \cdot\left\{(\beta+1) \exp \{-\alpha(\beta+1)\}+\frac{1}{\alpha}\left[-\alpha+\frac{2 \omega}{e t(1-p)}\right]\right\},
\end{aligned}
$$

where the last inequality of (A.23) follows from being (13) true.

Under the hypothesis that $\underline{\alpha}>(1-p) / 2$, the right-hand side of (A.23) is greater than 0 , and this gives the required thesis.

(ii) Formula (16) gives

$$
\begin{aligned}
\left|\mathcal{A}_{2}(\alpha, \beta)\right|= & \frac{\omega}{\operatorname{et}[1-\exp \{-\alpha(\beta+1)\}]} \cdot\left[1-\exp \{-\alpha(\beta+1)\}-\frac{\omega}{\operatorname{et}(1-p)}\right] \\
& +\exp \{-\alpha(\beta+1)\}+\alpha .
\end{aligned}
$$

Therefore,

$$
\frac{\partial\left|\mathcal{A}_{2}(\alpha, \beta)\right|}{\partial \alpha}=(\beta+1) \exp \{-\alpha(\beta+1)\} \cdot\left[\frac{2 \omega^{2}}{(e t)^{2}(1-p)(1-\exp \{-\alpha(\beta+1)\})^{2}}-1\right]+1 .
$$


A simple computation assures the following inequalities:

$$
\left\{\begin{array}{l}
\frac{2 \underline{\alpha}}{1-p}>\frac{2 \underline{\alpha}^{2}}{1-p} \\
(1-\exp \{-\alpha(\beta+1)\})^{2}<1 .
\end{array}\right.
$$

Thus, by the hypothesis, we have

$$
\frac{2 \omega^{2}}{(e t)^{2}(1-p)(1-\exp \{-\alpha(\beta+1)\})^{2}}-1>0,
$$

and this implies the thesis. 